\title{
A rare case of isolated non-typhoidal Salmonella lymphadenitis
}

\author{
Alice Behrens MD, Hans Wang MD, Kevin Kuriakose MD, Emily Behrens MD, Matthew Burns MD
}

\begin{abstract}
Over $95 \%$ of cases of typhoidal and non-typhoidal Salmonella infections are foodborne. Focal salmonellosis without bacteremia is rarely reported, especially cases of non-enteric lymphadenitis. We present a rare case of Salmonella typhimurium inguinal lymphadenitis and review major risk factors for focal salmonellosis and appropriate treatments.
\end{abstract}

Keywords: Salmonella typhimurium, lymphadenitis, fluoroquinolone

\section{INTRODUCTION}

Salmonellae are gram-negative facultative anaerobic bacilli. Over $95 \%$ of cases of Salmonella infections are foodborne. ${ }^{1}$ More than 2,500 Salmonella serotypes have been identified, which can be categorized into typhoidal and non-typhoidal salmonellosis. Non-typhoidal salmonellosis refer to illnesses caused by all serotypes of Salmonella, excluding Typhi, Paratyphi A, Paratyphi B (tartrate negative), and Paratyphi C. Non-typhoidal Salmonella may seed virtually any anatomical site hematogenously. ${ }^{2}$ To the best of our knowledge, only 5 cases of non-typhoidal Salmonella non-enteric lymphadenitis have been reported.

\section{CASE}

A 54-year-old African American veteran with history of type-2 diabetes mellitus, hypertension, hyperlipidemia, and gastro-esophageal reflux disease presented to the emergency department (ED) with a 1-day history of right inguinal pain and right lower quadrant

Corresponding author: Alice W. Behrens Contact Information: Abehrens@uams.edu DOI: 10.12746/swrccc.v7i30.534 abdominal pain. The patient complained of shooting pain radiating down to his right testicle which was worsened with coughing and a white penile discharge. He denied any injury, heavy lifting, fever, chills, nausea, vomiting, and diarrhea. He rarely goes outdoors and denied recent travel. He also denied recent tick bites and exposure to house pets and dead animals. His last sexual activity was months ago. He had a history of gonorrhea many years earlier, but no other sexually transmitted infections (STIs). His home mediations included metformin $1000 \mathrm{mg}$ twice a day and aspirin $81 \mathrm{mg}$ daily.

During his first ED visit, his vital signs were within normal limits. Physical examination was significant for a $7 \times 4 \mathrm{~cm}$ firm, exquisitely tender right inguinal mass, which was not warm to palpation. No testicular tenderness, urethral discharge, or erythema was noted. Laboratory values were significant for leukocytosis of 12,000 cells $/ \mu$ l with neutrophilic predominance. Computed tomography (CT) of the abdomen and pelvis revealed an enlarged inguinal node measuring up to $2.3 \mathrm{~cm}$ in diameter with phlegmonous changes (Figure 1A). No surgical drainage was performed. He was discharged on a 15-day course of oral doxycycline which he completed.

Three weeks after his initial presentation, he returned to the ED with 2-days of right inguinal pain and increased swelling that woke him up from sleep. 


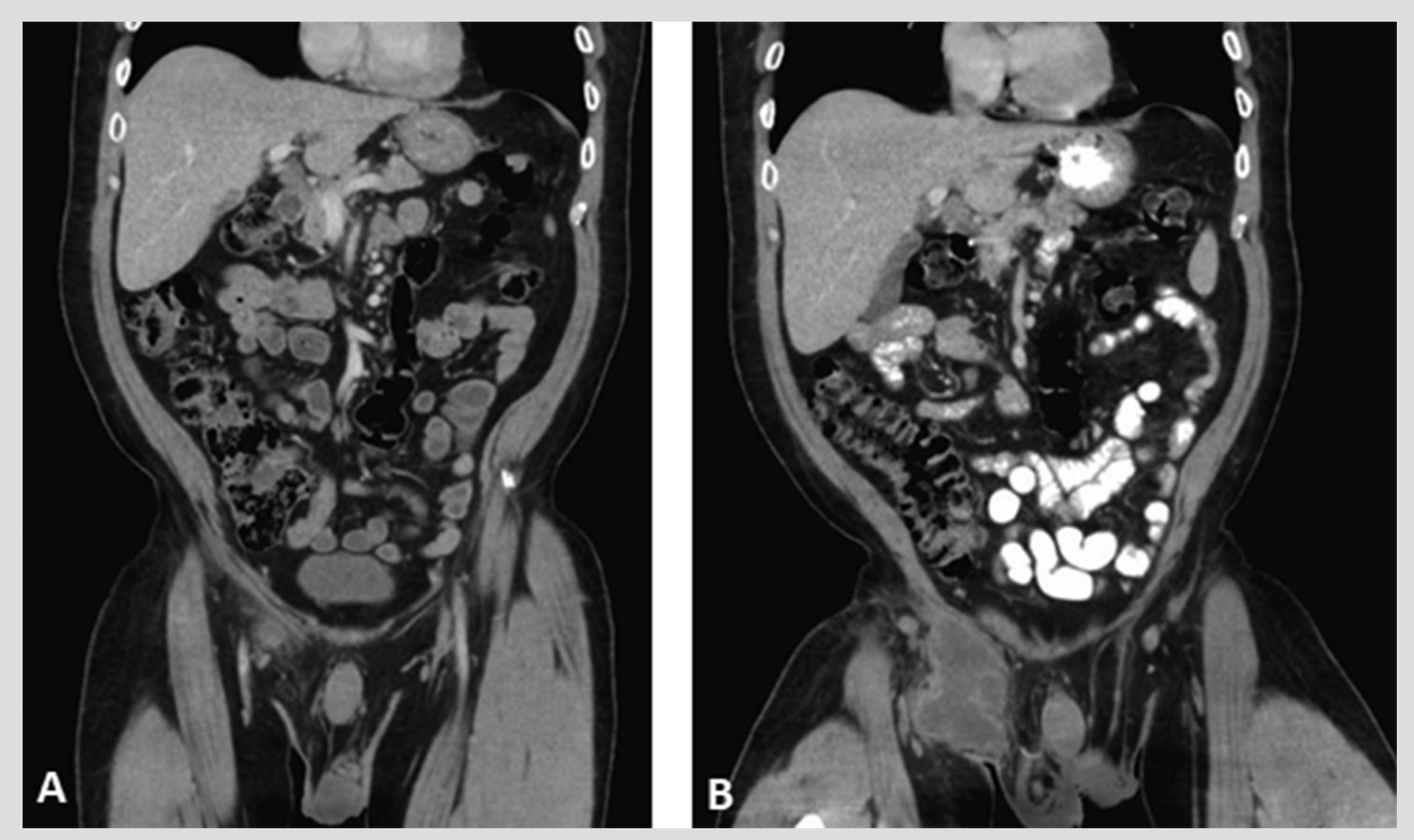

Figure 1. A) A CT scan of the abdomen/pelvis on the initial ED visit showing an enlarged partially hypodense right inguinal lymph node, measuring up to $2.3 \mathrm{~cm}$, with reactive inflammatory changes in the adjacent soft tissue. B) A CT scan of abdomen/pelvis on second ED visit 6 weeks later showing the right inguinal lymph node previously identified has had significant enlargement, now measuring $6.4 \times 4.1 \times 9.1 \mathrm{~cm}$.

He denied fever, chills, dysuria, suprapubic tenderness, and flank pain. A repeat CT of abdomen/pelvis revealed interval enlargement of the right inguinal node measuring $6.4 \times 4.1 \times 9.1 \mathrm{~cm}$ (Figure 1B). He was started on intravenous vancomycin and piperacillin/ tazobactam empirically. Bedside incision and drainage were performed on the enlarged inguinal mass. Approximately $150 \mathrm{ml}$ of purulent fluid was drained and sent for culture. Several loculations were broken up using hemostats. He started to improve clinically with daily wound care. Preliminary culture results were positive for Salmonella species. He declined HIV testing. He was discharged on a 10-day course of oral ciprofloxacin.

Culture of the purulent drainage result became available 3 weeks later, revealing 1+ Salmonella enterica serotype Typhimurium 14, 12 i1,2. Antimicrobial sensitivity to ciprofloxacin had a minimum inhibitory concentration of 0.032 . Trimethoprim-sulfamethoxazole (TMP-SMZ) tested sensitive by disc diffusion. The patient was subsequently lost to follow up.

\section{Discussion}

Non-typhoidal Salmonella lymphadenitis may develop as a local infection, even with treatment of bacteremia. The incidence of non-typhoid salmonellosis is estimated at over 2 million cases annually, but extraintestinal manifestations account for less than $1 \%$ of these cases. ${ }^{3}$ Focal salmonellosis is thought to be secondary to a brief episode of bacteremia after infection from the gastrointestinal tract. Salmonella species can live for unknown periods of time within macrophages, resulting in a continuous carrier state. ${ }^{2}$ 
However, the chronic carrier state is only well reported in the typhoidal Salmonella serotypes. Long-term carriage of non-typhoidal Salmonella serotypes has not been described. Even though symptoms usually last only a few days, infected adults can shed Salmonella in their feces for one month on average after initial infections; children under the age of 5 years can shed bacteria in their feces for an average of 7 weeks. ${ }^{4}$

Focal pyogenic Salmonella infection is far less common than systemic infection. ${ }^{5}$ According to a study series of 6,250 salmonellosis cases from 1981 to 1992 in India, only 100 patients had focal pyogenic infection. ${ }^{5}$ Among those 100 patients, only one had an infected inguinal node. ${ }^{5}$ Salmonella lymphadenitis is rarely reported in literature, and to the best of our knowledge, only 5 cases of non-typhoidal Salmonella non-enteric lymphadenitis have been reported. ${ }^{6-10}$ Campbell found a case of cervical lymphadenitis caused by Salmonella braenderup in a female with Hodgkin's lymphoma. ${ }^{6}$ Lim et al reported a case of cervical lymphadenitis caused by group $D$ non-typhoidal Salmonella associated with lymphoma in a 66-yearold woman. ${ }^{7}$ Pastelgia and Jenkins illustrated a case of submandibular lymphadenopathy caused by Salmonella typhimurium involving a para-pharyngeal space in a 55-year-old diabetic man. ${ }^{8}$ Cohen et al reported a case of Salmonella typhimurium axillary lymphadenitis with infiltration of a reticulum cell sarcoma in a 68-year-old diabetic patient. ${ }^{9}$ Dylewski et al described a previously healthy 10 -year-old boy developing submandibular granulomatous lymphadenitis caused by Salmonella serogroup B. ${ }^{10}$

The most common risk factors for developing salmonellosis include corticosteroid use, malignancy, diabetes, human immunodeficiency virus (HIV) infection, prior use of antimicrobial agents, and other immunosuppressive drugs. ${ }^{4}$ Anatomical disruptions, including kidney stones, other urinary tract abnormalities, gallstones, atherosclerotic endovascular lesions, and prosthetic devices, may all serve as foci for persistent Salmonella infection. ${ }^{1}$ In our patient, the only identifiable risk factor was his poorly controlled diabetes mellitus. The HIV status of this patient could not be determined as he declined testing. The source for this patient's isolated inguinal lymphadenitis is unclear given negative bacteremia and lack of Gl symptoms. We postulated that he may have developed transient asymptomatic intestinal non-typhoidal salmonellosis.

Tetracyclines generally do not have good activity against Salmonella. Antibiotics with good Salmonella coverage include fluoroquinolones, TMP-SMZ, ampicillin, or a third-generation cephalosporin. ${ }^{2}$ Because of the increasing resistance to TMP-SMZ and ampicillin, third-generation cephalosporins or quinolones are reasonable alternatives if susceptibilities are not yet available. ${ }^{2} \mathrm{~A}$ minimum of 2 weeks of antimicrobial therapy is suggested for the treatment of a surgically eradicated soft-tissue focus in a normal patient. ${ }^{1}$

Untreated Salmonella bacteremia in adults can lead to the development of infectious endarteritis, especially involving the abdominal aorta. ${ }^{9} \mathrm{~A}$ case study by Cohen et al in 1978 found that $25 \%$ of bacteremic adults over 50 years of age, identified by positive results of blood cultures, developed arteritis or endocarditis. ${ }^{9}$ For patients with painful focal lymphadenitis caused by gram-negative motile bacilli with a history of STIs, the differential diagnosis includes glandular tularemia, chancroid, and lymphogranuloma venereum. Although Salmonella is not a common cause, given the possible serious systemic complications from typhoidal and non-typhoidal Salmonella, clinicians may need to consider salmonellosis in their differential diagnoses and antimicrobial coverage.

Article citation: Behrens A, Wang $\mathrm{H}$, Kuriakose $\mathrm{K}$, Behrens E, Burns M. A rare case of isolated non-typhoidal Salmonella lymphadenitis. The Southwest Respiratory and Critical Care Chronicles 2019;7(30):54-57

From: Division of Infectious Disease (AB, HW, KK, MB), Department of Internal Medicine, University of Arkansas for Medical Sciences, Little Rock, AR; Department of Dermatology (EB), Texas Tech University Health Sciences Centers, Lubbock, TX.

Submitted: $5 / 9 / 2019$

Accepted: 6/15/2019

Reviewer: David Sotello MD

Conflicts of interest: none

This work is licensed under a Creative Commons Attribution-ShareAlike 4.0 International License. 


\section{REFERENCES}

1. Hohmann EL. Nontypohoidal salmonellosis. Clin Infect Dis 2001;32(2):263-269.

2. Gal-Mor O, Boyle EC, Grassl GA. Same species, different diseases: how and why typhoidal and non-typhoidal Salmonella enterica serovars differ. Front Micro 2014;5:391.

3. Kwon MH., Kang MI, Chun JY, et al. A case of neck abscess caused by Salmonella serotype D in a patient with liver cirrosis. Yonsei Med J 2010;5:128-130.

4. Chen PL, Chang CM, Wu CJ, et al. Extraintestinal focal infections in adults with nontyphoid Salmonella bacteraemia: predisposing factors and clinical outcome. J Intern Med 2007;261:91-100.

5. Lalitha MK, John R. Unusual manifestation of salmonellosis - a surgical problem. Q J Med 1994;87(5):301-9.
6. Campbell WN. Salmonella lymphadenitis associated with undiagnosed lymphoma. Eur J Clin Micro Infect Dis 2001 (20)5:359-361

7. Lim S, Cho SY, Kim J, et al. Cervical lymphadenitis caused by group D non-typhoidal Salmonella associated with concomitant lymphoma. Infect Chemother 2013;45(2): 234-238.

8. Pastagia M, Jenkin, SG. Salmonella neck abscess as an opportunistic infection in diabetes mellitus. Case Reports Infect Dis 2013. Doi: http://dx.doi.org/10.1155/2013/708419

9. Cohen JL, Bartlett JA, Corey GR: Extraintestinal manifestations of Salmonella infections. Medicine 01 Sep 1987; 66(5):349-388.

10. Dylewski JS, Roy I, Libman M. Granulomatous submandibular lymphadenitis in a healthy child. Clin Infect Dis 1994; 19:1175-1176. 